\title{
openheart Routine diversion of patients with STEMI to high-volume PCI centres: modelling the financial impact on referral hospitals
}

\author{
Elizabeth Barnett Pathak, ${ }^{1}$ Meg M Comins, ${ }^{2}$ Colin J Forsyth, ${ }^{3}$ Joel A Strom ${ }^{4}$
}

To cite: Pathak EB, Comins MM, Forsyth CJ, et al. Routine diversion of patients with STEMI to highvolume PCl centres: modelling the financial impact on referral hospitals. Open Heart 2015;2:e00042 doi:10.1136/openhrt-2014000042

Received 17 January 2014 Revised 24 June 2014 Accepted 5 June 2015

CrossMark

\begin{abstract}
${ }^{1}$ Department of Internal Medicine, University of South Florida, Tampa, Florida, USA ${ }^{2}$ Department of Health Policy and Management, University of South Florida, Tampa, Florida, USA

${ }^{3}$ Department of

Anthropology, University of South Florida, Tampa,

Florida, USA

${ }^{4}$ Department of Medicine, University of Florida College of Medicine, Jacksonville, Florida, USA
\end{abstract}

\section{Correspondence to} Dr Elizabeth Barnett Pathak; dr.elizabeth.pathak@gmail. com

\section{ABSTRACT}

Objective: To quantify possible revenue losses from proposed ST-elevation myocardial infarction (STEMI) patient diversion policies for small hospitals that lack high-volume percutaneous coronary intervention (PCI) capability status (ie, 'STEMI referral hospitals').

Background: Negative financial impacts on STEMI referral hospitals have been discussed as an important barrier to implementing regional STEMI bypass/transfer protocols. However, there is little empirical data available that directly quantifies this potential financial impact.

Methods: Using detailed financial charges from Florida hospital discharge data, we examined the potential negative financial impact on 112 STEMI referral hospitals from losing all inpatient STEMI revenue. The main outcome was projected revenue loss (PRL), defined as total annual patient with STEMI charges as a proportion of total annual charges for all patients. We hypothesised that for most community hospitals $(>90 \%)$, STEMI revenue represented only a small fraction of total revenue $(<1 \%)$. We further examined the financial impact of the 'worst case' scenario of loss of all acute coronary syndrome (ACS) (ie, chest pain) patients.

Results: PRLs were $\$ 0.33$ for every $\$ 100$ of patient revenue statewide for STEMI and $\$ 1.73$ for ACS. At the individual hospital level, the 90th centile PRL was $\$ 0.74$ for STEMI and \$2.77 for ACS. PRLs for STEMI were not greater in rural areas compared with major metropolitan areas. Hospital revenue centres that would be most impacted by loss of patients with STEMI were cardiology procedures and intensive care units.

Conclusions: Loss of patient with STEMI revenues would result in only a small financial impact on STEMI referral hospitals in Florida under proposed STEMI diversion/rapid transfer protocols. However, spillover loss of patients with ACS would increase revenue loss for many hospitals.

\section{INTRODUCTION}

Coronary heart disease continues to be the leading cause of death in the USA, and an estimated 525000 new and 210000 recurrent

\section{KEY MESSAGES}

What is already known about this subject?

- To our knowledge, this is the first study to directly study the financial impact of transfer of ST-elevation myocardial infarction (STEMI) patients on the small non-percutaneous coronary intervention (PCI)-providing hospitals (ie, 'STEMI referral centres').

\section{What does this study add?}

- Our results show that the vast majority of STEMI referral centres will experience only a very small financial impact from the loss of patients with STEMI through evidence-based transfer to a high-volume $\mathrm{PCI}$ center.

How might this impact on clinical practice?

- Cooperation between high-volume PCl-providing hospitals and STEMI referral centers is essential to ensure optimal clinical care. Numerous barriers to timely transfer of patients with STEMI may exist in these smaller community hospitals. Knowledge that hospital finances will not be adversely impacted by 'giving away' patients with STEMI can empower hospital physicians.

myocardial infarctions (MIs) will occur in 2015. ${ }^{1}$ Rapid access to primary percutaneous coronary intervention (PCI) for ST-segment elevated myocardial infarction (STEMI) patients results in earlier myocardial reperfusion that improves short and long-term outcomes. $^{2-9}$ However, only about $20 \%$ of acute care hospitals in the USA have cardiac catheterisation laboratories, ${ }^{10}$ and only a subset of these hospitals offer primary PCI on a fulltime basis. ${ }^{10}$ Additionally, PCI hospitals are more likely to be located in large urban centres, leaving many rural areas without access to PCI. ${ }^{11}$

Consequently, the American Heart Association, as part of its own initiative, sponsored a conference in early 2006 on development of systems of care for patients with STEMI. ${ }^{12}$ Barriers to implementing STEMI 
systems of care were examined from the perspectives of non-PCI hospitals, ${ }^{13}$ PCI-capable hospitals, ${ }^{14}$ emergency services, ${ }^{15}$ physicians, ${ }^{16}$ patients ${ }^{17}$ and payers. ${ }^{18}$ In an ideal system, emergency medical services (EMS) personnel would bypass non-PCI capable hospitals and transport patients directly to a high-volume PCI centre. In some cases, non-PCI-capable hospital bypass would be not feasible or patients self-transport to non-PCI capable hospitals, but hospital transfer could still result in PCI within $120 \mathrm{~min}$ of first medical contact. ${ }^{19}$ In these cases, ECG diagnosis of STEMI would be performed by EMS personnel prior to emergency department (ED) arrival, or by ED personnel within $10 \mathrm{~min}$ of arrival; patients would then be emergently transported to a high-volume PCI centre within $30 \mathrm{~min}^{13}$ Clinical trials in Europe $^{8} 9^{20-22}$ and a recent trial in the $\mathrm{USA}^{23}$ have shown that the routine transfer of patients with STEMI to PCI centres is safe and feasible, and can be accomplished rapidly within recommended time-to-reperfusion guidelines.

Despite this persuasive evidence, controversy exists in the USA about the potential for implementing routine regional STEMI bypass/transfer protocols. A major concern for both non-PCI capable and low-volume PCI hospitals is the possible negative financial impact from losing patients to high-volume PCI centres. ${ }^{12}{ }^{13} \quad 15$ Recent policy statements have made dramatic statements about the detriment to these hospitals, for example: “.... their very survival may be threatened." ${ }^{13}$ However, there is little empirical data available that directly quantifies this potential financial impact.

In this study, we analysed empirical data for all patients with STEMI admitted to acute-care general hospitals in Florida and statistically modelled the potential negative financial impact on non-PCI capable hospitals and lowvolume PCI hospitals of losing all inpatient patient with STEMI revenue as result of the proposed regionalisation plans that would either bypass those hospitals or require rapid transfer protocols. Contrary to some of the prevailing opinion, we hypothesised that for most community hospitals $(>90 \%)$, STEMI revenue represented only a small fraction of total revenue $(<1 \%)$ and consequently loss of those patients would not threaten hospital viability. We further examined whether the proportion of revenue from patients with STEMI varied by rurality, hospital characteristics (PCI volume and STEMI volume), hospital revenue centre (eg, intensive care unit) or patient insurance type. Finally, a second set of analyses modelled the financial impact of loss of all acute coronary syndrome (ACS) patient revenue, given arguments that STEMI referrals would lead to a wider loss of chest pain patients to high-volume PCI centres.

\section{METHODS}

\section{Study population and data sources}

Our study population consisted of adults aged 18 years and above who were discharged from Florida acute care general hospitals with a primary diagnosis of STEMI during 2006. Our primary data source was the Florida Agency for Health Care Administration (AHCA) Hospital Discharge Database. All hospitals in Florida (except VA/Federal hospitals) are required by state law to submit a mandatory dataset each year to AHCA. These data include all hospital discharge records, and therefore do not represent a sample, but rather surveillance with $100 \%$ coverage. AHCA makes available public-use data sets that have been de-identified for patients (but not for hospitals). Each discharge record contained patient-level data on source and day of admission, demographics, insurance status/payer, primary and secondary diagnoses (up to 30), primary and secondary procedures (up to 30), length of stay, discharge destination (including vital status at discharge) and financial charges for 22 specific revenue groups. Diagnoses and procedures were recorded using detailed codes from the International Classification of Diseases, Ninth Revision, Clinical Modification (ICD-9-CM). Hospitals were identified by a unique numeric identification number. Additional data on hospital and community characteristics were obtained from the American Hospital Association Guide ${ }^{24}$ and from the US Census of Population and Housing. ${ }^{25}$

In this paper, we use the term 'STEMI referral hospitals' to refer to hospitals that were non-PCI capable (no PCI procedures performed on inpatients) or were lowvolume PCI centres (fewer than 200 PCI procedures performed annually). The American College of Cardiology/ American Heart Association (ACC/AHA) practice guidelines for PCI designate 'high-volume' centres as those hospitals that perform $400+$ PCIs on an annual basis. ${ }^{26}$ We calculated hospital PCI volumes by summing the total number of procedures at each hospital, regardless of principal diagnosis. Patients with STEMI were identified by the principal diagnosis field on the discharge record, based on ICD-9-CM codes (410.0-410.6 and 410.8). Patients with ACS were identified by principal diagnosis codes 410 (STEMI, NSTEMI, and unspecified acute myocardial infarction (AMI)) and 411 (unstable angina).

\section{Financial analyses}

The main outcome in this study was projected revenue loss (PRL), defined as total annual patient with STEMI charges as a proportion of total annual charges for all patients. For each individual hospital, we multiplied the raw charge data by actual cost-to-charge ratios to obtain cost estimates, which were then used to provide the best estimates of PRL. This was a necessary step because in the USA, hospital charges are often inflated values. The ratio of actual costs to reported financial charges varies within hospitals (by department) and also between hospitals. Medicare Healthcare Cost Report Information System data were used to calculate cost-to-charge ratios at the department level. Hospital systems often report many hospitals under one Medicare Provider Number (MPN). In these instances, the cost ratios reported 
under one MPN were applied to each hospital within that system. For the analyses of specific hospital revenue centres, we chose cardiology, intensive care unit, radiology and pharmacy due to their relevance to patients with STEMI and ACS. Under a 'worst case' hypothetical scenario, we assumed that all STEMI inpatient revenue would be lost, and calculated the per cent reduction in total revenue that would result. We first calculated this measure for all STEMI referral hospitals across the state as a whole, and then for several statewide strata: community rurality index, hospital type, hospital revenue centre and payer. We then calculated the STEMI PRL for each individual hospital, and examined the distribution of PRL estimates in accordance with our primary hypothesis. 95\% CIs were calculated for all PRLs. Hospital-level cost-to-charge ratios were used to adjust the PRL estimates for all strata except for revenue centres, for example, cardiology procedures, where for an accurate PRL calculation the department-level cost-to-charge ratios were used. ${ }^{27}$

To calculate the PRL, adjusted charges for all hospital inpatients regardless of diagnosis were summed to form the denominator. The sum of all STEMI patient-adjusted charges formed the numerator. For the hospital revenue centre analyses, we grouped financial charges data into four categories (from 22 categories provided on the discharge record). These categories were: (1) cardiology procedures; (2) intensive or coronary care unit; (3) radiology; (4) pharmacy. Not all hospitals had charges in each revenue centre. We also examined six major payer types: (1) Medicare, including HMO; (2) Medicaid, including HMO; (3) commercial; (4) self-pay/underinsured; (5) charity; (6) other (eg, Tricare military insurance, worker's compensation). There were $\$ 0$ charges for charity patients at 33 hospitals (meaning these hospitals did not report charges classified as charity; they may have been reported with self-pay).

Our secondary financial analyses focused on PRL from all ACS and followed the same methods outlined above for STEMI.

\section{Transfer time analyses}

We used hospital street addresses and geographic information systems methods to calculate road network travel distances and transport time from each STEMI referral hospital to the nearest high-volume PCI centre, for the subset of hospitals whose annual PRL for STEMI was $>1 \%$ ( $>\$ 1$ per $\$ 100$ of total patient revenue).

\section{Exclusions}

We excluded certain hospital types from our analyses: children's hospitals, psychiatric and behavioural health facilities, and long-term care facilities. Data were not available for Veteran's Administration facilities which may infrequently accept patients with AMI through their EDs. We also excluded eligible acute care community hospitals which did not discharge any patients with STEMI during the study period $(n=6)$. Patients were excluded only on the basis of age $(<18$ years of age). We did not exclude either 'transfers in' or 'transfers out' to/from other acute care hospitals, in order to maximise the 'worst case scenario' approach to examining our primary hypothesis-in other words, to maximise the estimate of projected revenue lost. Three critical access hospitals $^{28}$ with a total of 21 STEMI discharges did not report costs to Medicare; therefore cost-to-charge ratios for these hospitals could not be calculated and they were excluded from all analyses.

\section{RESULTS \\ PRL for STEMI}

In this study, 2082 patients with STEMI were admitted to 112 STEMI referral hospitals with an annual PCI volume of $<200$ procedures/year (table 1 ). The typical patient was a white male admitted to the hospital through the emergency department. The most common age group was $65-84$ years $(44.4 \%)$, with $31.2 \%$ of patients $<65$ years and $19.4 \%$ of patients $>85$ years of age. Over $90 \%$ of patients had some type of health insurance, with $2.3 \%$ identified as charity patients and $7.2 \%$ as self-pay/underinsured. Almost $70 \%$ of these patients had a length of stay $>2$ days. Among the subset of patients who were eventually transferred to another (presumably larger) hospital, almost $50 \%$ were not transferred until 2+ days after admission.

Characteristics of the STEMI referral hospitals are shown in table 2. The majority of these hospitals performed no PCIs $(69.6 \%)$, and $93 \%$ admitted $<51$ patients with STEMI-equivalent to $<1$ patient with STEMI per week on average. According to self-reports in an American Hospital Association survey, 26.8\% offered diagnostic cardiac catheterisation and $44.6 \%$ had a medical intensive care unit (MICU); however, $42.6 \%$ of hospitals did not participate in the survey. Almost half of all hospitals were for-profit $(49.1 \%)$ and the majority were located in a medium or large metropolitan area $(76.7 \%)$, that is, in an urban area with a population of at least 250000 . Only 10 STEMI referral hospitals were located in rural areas or small towns.

We first examined average PRL for STEMI for the state as a whole (table 3). Across all 112 STEMI referral hospitals which admitted at least 1 patient with STEMI annually, the annual PRL was $\$ 0.33$ for every $\$ 100$ of total patient revenue. We then divided the hospitals into groups based on specific hospital characteristics and calculated annual PRLs for each group. The PRL for rural areas $(\$ 0.24)$ was lower than the PRL for major metro areas $(\$ 0.33)$. The average PRL was greatest among hospitals with the highest PCI volume $(\$ 0.96)$ and highest patient with STEMI volume (\$1.08). Average PRLs varied by hospital revenue centre, with the highest average PRL for cardiology services (\$2.27). Average PRL was higher for Medicare patients $(\$ 0.36)$ than for commercial patients $(\$ 0.30)$.

Annual STEMI PRLs for individual hospitals ranged from $\$ 0.01$ to $\$ 3.82$ (table 4). The PRL at the 90th centile was $\$ 0.74 / \$ 100$ of total patient revenue. The 
Table 1 Characteristics of STEMI $(n=2082)$ and ACS patients $(n=12228)$ at STEMI referral hospitals* in Florida

\begin{tabular}{lcc}
\hline Patient characteristics & $\begin{array}{l}\text { STEMI } \\
\text { Per cent } \\
\text { (number) }\end{array}$ & $\begin{array}{l}\text { ACS } \\
\text { Per cent } \\
\text { (number) }\end{array}$ \\
\hline Admission source & & \\
Emergency department & $94.1(1959)$ & $95.2(11644)$ \\
Direct admission & $5.5(114)$ & $4.3(529)$ \\
Transfer int & $0.4(8)$ & $0.3(34)$ \\
Other & $0.1(1)$ & $0.2(21)$ \\
Age (years) & & \\
18-44 & $5.0(104)$ & $3.8(469)$ \\
45-64 & $31.2(649)$ & $24.0(2933)$ \\
65-84 & $44.4(925)$ & $49.4(6038)$ \\
85+ & $19.4(404)$ & $22.8(2788)$ \\
Gender & & \\
Male & $58.7(1221)$ & $55.1(6740)$ \\
Female & $41.3(861)$ & $44.9(5488)$ \\
Race/ethnicity & & \\
White & $79.5(1656)$ & $79.6(9737)$ \\
Hispanic & $10.5(219)$ & $9.1(1118)$ \\
Black & $6.6(138)$ & $8.6(1055)$ \\
Other & $3.4(69)$ & $2.7(318)$ \\
Insurance type & & \\
Medicare & $61.5(1280)$ & $70.4(8609)$ \\
Medicaid & $5.3(110)$ & $4.3(524)$ \\
Commercial & $22.8(474)$ & $16.7(2045)$ \\
Self pay/underinsured & $7.2(151)$ & $5.7(696)$ \\
Charity & $2.3(47)$ & $1.7(211)$ \\
Other & $1.0(20)$ & $1.2(143)$ \\
Length of hospital stay (no transfer) (days) & \\
1 & $31.0(645)$ & $24.2(2961)$ \\
2 & $15.3(319)$ & $14.9(1825)$ \\
3-6 & $38.5(801)$ & $41.0(5014)$ \\
7+ & $15.2(317)$ & $19.9(2428)$ \\
Length of hosptal & &
\end{tabular}

Length of hospital stay (prior to transfer to another hospital) (days)

$\begin{array}{lcc}1 & 51.5(514) & 45.1(2387) \\ 2 & 19.6(196) & 20.2(1072) \\ 3-6 & 24.8(247) & 28.9(1529) \\ 7+ & 4.1(41) & 5.9(310)\end{array}$

*Includes hospitals with $>1$ patient with STEMI discharged and $<200 \mathrm{PCl}$ s performed in 2006.

†Transfer in from another short-term acute care hospital. ACS, acute coronary syndrome; $\mathrm{PCl}$, percutaneous coronary intervention; STEMI, ST-elevation myocardial infarction.

90th centile PRL was less than $1 \%(\$ 1)$ in all categories of urbanicity/rurality. However, the 90th centile PRL exceeded $1 \%$ for the four hospitals with highest PCI volume (\$3.82) and for the eight hospitals with highest STEMI volume (\$3.82).

\section{PRL for ACS}

We examined PRL for ACS under assumption of a "worst case' scenario in which STEMI diversion/rapid transfer protocols would result in the spillover loss of all acute chest pain patients from STEMI referral hospitals. There were 12228 patients with ACS admitted to STEMI referral hospitals in Florida during the study period
Table 2 Characteristics of STEMI referral hospitals* $(n=112)$ in Florida

\begin{tabular}{lc}
\hline Hospital characteristics & Per cent \\
(number)
\end{tabular}

*Includes hospitals with $>1$ patient with STEMI discharged and $<200 \mathrm{PCls}$ performed in 2006.

$\mathrm{PCl}$, percutaneous coronary intervention; STEMI, ST-elevation myocardial infarction.

(table 1). Average annual PRL for ACS was over five times higher than the PRL for STEMI alone ( $\$ 1.73$ vs $\$ 0.32$ ) but still $<2 \%$ (table 3). Average annual PRLs exceeded $2 \%$ for hospitals with highest PCI volume (\$2.53) and highest STEMI volume (\$2.84), for Medicare patients $(\$ 2.24)$ and for the following specific revenue centres: cardiology (\$8.93) and MICU/cardiac care unit (CCU) (\$3.45). For individual hospitals, the 90th centile value of PRL for ACS was $\$ 2.77$ (table 5). The median (50th centile) value was $\$ 1.49$, indicating that $>50 \%$ of these hospitals would experience revenue loss of $>1 \%$ if all patients with ACS were lost. Median PRLs were highest for the four hospitals with highest PCI volume (\$2.53) and for the eight hospitals with highest STEMI volume (\$2.89). Interestingly, the 90th centile PRL for cardiology services was $\$ 14.42$-indicating that non-ACS patients accounted for $>86 \%$ of cardiology revenue in the majority of these STEMI referral hospitals. 
Table 3 PRLs resulting from proposed diversion of STEMI patients and ACS* patients away from referral hospitals with low or no PCI capability: summary results

\begin{tabular}{|c|c|c|c|c|c|}
\hline $\begin{array}{l}\text { Summary level for } \\
\text { community hospital } \\
\text { financial data }\end{array}$ & $\begin{array}{l}\text { Patients with } \\
\text { STEMI total } \\
\text { charges } \dagger(n=2082)\end{array}$ & $\begin{array}{l}\text { All patients with } \\
\text { ACS total chargest } \\
(n=12228)\end{array}$ & $\begin{array}{l}\text { All patients } \\
\text { total charges } t \\
(n=814831)\end{array}$ & $\begin{array}{l}\text { Annual projected loss } \\
\text { per } \$ 100 \text { of total revenue } \\
\text { Patients with STEMI }\end{array}$ & $\begin{array}{l}\text { Annual projected loss } \\
\text { per } \$ 100 \text { of total revenue } \\
\text { ACS }^{*} \text { patients }\end{array}$ \\
\hline Florida totals & $\$ 16500$ & $\$ 86494$ & $\$ 5004176$ & $\$ 0.33(\mathrm{Cl} \$ 0.33$ to $\$ 0.33)$ & $\$ 1.73(\mathrm{Cl} \$ 1.73$ to $\$ 1.73)$ \\
\hline \multicolumn{6}{|l|}{ Community location } \\
\hline Rural/non-metro & $\$ 251$ & $\$ 2017$ & $\$ 102420$ & $\$ 0.24(\mathrm{Cl} 0.24$ to 0.25$)$ & $\$ 1.97(\mathrm{Cl} 1.97$ to 1.97$)$ \\
\hline Small metro/urban & 1116 & 8052 & 531311 & $0.21(\mathrm{Cl} 0.21$ to 0.21$)$ & $1.51(\mathrm{Cl} 1.51$ to 1.52$)$ \\
\hline Medium metro & 3328 & 15742 & 837840 & $0.40(\mathrm{Cl} 0.40$ to 0.40$)$ & $1.88(\mathrm{Cl} 1.88$ to 1.88$)$ \\
\hline Major metro & 11806 & 60681 & 3532604 & $0.33(\mathrm{Cl} 0.33$ to 0.33$)$ & $1.72(\mathrm{Cl} 1.72$ to 1.72$)$ \\
\hline \multicolumn{6}{|l|}{ PCl capability in 2006} \\
\hline None performed & $\$ 8964$ & $\$ 52743$ & $\$ 3170838$ & $\$ 0.28(\mathrm{Cl} 0.28$ to 0.28$)$ & $\$ 1.66(\mathrm{Cl} 1.66$ to 1.66$)$ \\
\hline $1-11$ & 2758 & 20474 & 1248034 & $0.22(\mathrm{Cl} 0.22$ to 0.22$)$ & $1.64(\mathrm{Cl} 1.64$ to 1.64$)$ \\
\hline $12-51$ & 519 & 2003 & 140423 & $0.37(\mathrm{Cl} 0.37$ to 0.37$)$ & $1.42(\mathrm{Cl} 1.42$ to 1.43$)$ \\
\hline $52+$ & 4259 & 11273 & 444880 & $0.96(\mathrm{Cl} 0.96$ to 0.96$)$ & $2.53(\mathrm{Cl} 2.53$ to 2.53$)$ \\
\hline \multicolumn{6}{|l|}{ Patients with STEMI in 2006} \\
\hline $1-11$ & $\$ 2020$ & $\$ 19270$ & $\$ 1728659$ & $\$ 0.12(\mathrm{Cl} 0.12$ to 0.12$)$ & $\$ 1.11(\mathrm{Cl} 1.11$ to 1.11$)$ \\
\hline $12-51$ & 8384 & 51246 & $\$ 2712559$ & $0.31(\mathrm{Cl} 0.31$ to 0.31$)$ & $1.89(\mathrm{Cl} 1.89$ to 1.88$)$ \\
\hline $52+$ & 6096 & 15977 & 562958 & $1.08(\mathrm{Cl} 1.08$ to 1.08$)$ & $2.84(\mathrm{Cl} 2.84$ to 2.84$)$ \\
\hline \multicolumn{6}{|l|}{ Hospital revenue center } \\
\hline Cardiology procedures§ & 1683 & 6608 & 74013 & $2.27(\mathrm{Cl} 2.27$ to 2.28$)$ & $8.93(\mathrm{Cl} 8.92$ to 8.93$)$ \\
\hline $\mathrm{MICU} / \mathrm{CCU}$ & 7410 & 43336 & 1254309 & $0.59(\mathrm{Cl} 0.59$ to 0.59$)$ & $3.45(\mathrm{Cl} 3.45$ to 3.46$)$ \\
\hline Radiology & 572 & 3805 & 326920 & $0.17(\mathrm{Cl} 0.17$ to 0.18$)$ & $1.16(\mathrm{Cl} 1.16$ to 1.17$)$ \\
\hline Pharmacy & 2213 & 10,464 & 579701 & $0.38(\mathrm{Cl} 0.38$ to 0.38$)$ & $1.81(\mathrm{Cl} 1.80$ to 1.81$)$ \\
\hline \multicolumn{6}{|l|}{ Patients by payer } \\
\hline Medicare & $\$ 10150$ & $\$ 62336$ & $\$ 2785263$ & $\$ 0.36(\mathrm{Cl} 0.36$ to 0.36$)$ & $\$ 2.24(\mathrm{Cl} 2.24$ to 2.24$)$ \\
\hline Medicaid & 1224 & 4800 & 534937 & $0.23(\mathrm{Cl} 0.23$ to 0.23$)$ & $0.90(\mathrm{Cl} 0.90$ to 0.90$)$ \\
\hline Commercia & 3641 & 12775 & 1200474 & $0.30(\mathrm{Cl} 0.30$ to 0.30$)$ & $1.06(\mathrm{Cl} 1.06$ to 1.07$)$ \\
\hline Self-pay/underinsured & 1051 & 4441 & 288755 & $0.36(\mathrm{Cl} 0.36$ to 0.36$)$ & $1.54(\mathrm{Cl} 1.54$ to 1.54$)$ \\
\hline Charity & 317 & 1450 & 105193 & $0.30(\mathrm{Cl} 0.30$ to 0.30$)$ & $1.38(\mathrm{Cl} 1.38$ to 1.38$)$ \\
\hline Other & 117 & 691 & 89553 & $0.13(\mathrm{Cl} 0.13$ to 0.13$)$ & $0.77(\mathrm{Cl} 0.77$ to 0.77$)$ \\
\hline
\end{tabular}


Table 4 PRLs resulting from proposed diversion of STEMI patients away from referral hospitals with low or no PCI capability: individual hospital results

\begin{tabular}{|c|c|c|c|c|c|c|}
\hline \multirow{2}{*}{$\begin{array}{l}\text { Summary level for community hospital } \\
\text { financial data ( } n=\text { number of hospitals) }\end{array}$} & \multicolumn{6}{|c|}{ Annual PRL (\%) } \\
\hline & Minimum & Median & Mean & 90th centile & 99th centile & Maximum \\
\hline All hospitals $(n=112)$ & 0.01 & 0.20 & 0.33 & 0.74 & 1.68 & 3.82 \\
\hline \multicolumn{7}{|l|}{ Community location } \\
\hline Small urban/rural $(n=10)$ & 0.05 & 0.22 & 0.29 & 0.66 & 0.93 & 0.93 \\
\hline Small rural/large urban $(n=16)$ & 0.02 & 0.11 & 0.20 & 0.48 & 0.72 & 0.72 \\
\hline Medium metropolitan $(n=22)$ & 0.04 & 0.27 & 0.40 & 0.94 & 1.68 & 1.68 \\
\hline Major metropolitan $(n=64)$ & 0.01 & 0.20 & 0.33 & 0.74 & 3.82 & 3.82 \\
\hline \multicolumn{7}{|l|}{$\mathrm{PCl}$ capability in 2006} \\
\hline None performed $(n=78)$ & 0.01 & 0.22 & 0.29 & 0.71 & 1.19 & 1.19 \\
\hline $1-11(n=26)$ & 0.01 & 0.11 & 0.25 & 0.72 & 1.68 & 1.68 \\
\hline $12-51(n=3)$ & 0.08 & 0.38 & 0.34 & 0.57 & 0.57 & 0.57 \\
\hline $52+(n=5)$ & 0.19 & 0.78 & 1.29 & 3.82 & 3.82 & 3.82 \\
\hline \multicolumn{7}{|l|}{ Patients with STEMI in 2006} \\
\hline $1-11(n=53)$ & 0.01 & 0.09 & 0.15 & 0.38 & 0.93 & 0.93 \\
\hline $12-51(n=51)$ & 0.06 & 0.30 & 0.35 & 0.71 & 1.03 & 1.03 \\
\hline $52+(n=8)$ & 0.43 & 0.99 & 1.31 & 3.82 & 3.82 & 3.82 \\
\hline \multicolumn{7}{|l|}{ Hospital revenue center ${ }^{*}$} \\
\hline Cardiology procedurest $(n=94 / 100)$ & 0.00 & 0.70 & 1.65 & 3.28 & 18.99 & 25.99 \\
\hline Intensive or coronary care unit $(n=105 / 107)$ & 0.00 & 0.44 & 0.64 & 1.36 & 3.13 & 4.31 \\
\hline Radiology $(n=112 / 112)$ & 0.00 & 0.14 & 0.19 & 0.40 & 0.77 & 1.21 \\
\hline Pharmacy $(n=112 / 112)$ & 0.01 & 0.22 & 0.40 & 0.86 & 2.82 & 3.66 \\
\hline \multicolumn{7}{|l|}{ Patient payment type* } \\
\hline Medicare patients $(n=110 / 112)$ & 0.02 & 0.27 & 0.37 & 0.75 & 1.55 & 3.93 \\
\hline Medicaid patients $(n=47 / 112)$ & 0.02 & 0.33 & 0.48 & 1.27 & 2.71 & 2.71 \\
\hline Commercial payer patients $(n=86 / 112)$ & 0.01 & 0.18 & 0.40 & 0.93 & 3.77 & 3.77 \\
\hline Self-Pay/under-insured patients $(n=55 / 112)$ & 0.07 & 0.46 & 1.08 & 2.05 & 11.89 & 11.89 \\
\hline Charity patients $(n=25 / 79)$ & 0.06 & 0.47 & 1.21 & 2.62 & 8.83 & 8.83 \\
\hline Other $(n=17 / 110)$ & 0.05 & 0.78 & 1.31 & 5.46 & 5.90 & 5.90 \\
\hline
\end{tabular}

\section{Hospitals with high STEMI PRLs}

Of 112 referral hospitals which discharged one or more patients with STEMI, there were five hospitals with STEMI PRLs $>1 \%$ (table 6). All were located in metropolitan areas of $>250000$ population, and all were less than a 31 min drive to the nearest high volume PCI centre. Hospitals $\mathrm{C}$ and $\mathrm{E}$ were unprofitable non-PCI capable centres with sizeable patient with STEMI volumes, located in very close proximity to high-volume PCI centres.

\section{DISCUSSION}

We found an average PRL of $0.33 \%$ for the entire state and for individual hospitals, a 90th centile PRL of $0.74 \%$, confirming our hypothesis that STEMI charges represented $<1 \%$ of total charges for more than $90 \%$ of Florida community hospitals. As expected, PRL was highest $(0.96 \%)$ in low-volume PCI hospitals which still performed more than 52 PCIs in 2006. Nonetheless, this subset consisted of only four hospitals, or $3.5 \%$ of the total sample. The lowest PRL of $0.22 \%$ occurred in the 14 hospitals which performed between 1 and 11 PCIs in
2006, while the PRL averaged $0.28 \%$ in the 78 hospitals which did not perform any PCIs in 2006.

Some fear that the implementation of regional STEMI care systems incorporating bypass/transfer protocols for PCI would irrevocably damage the financial viability of community hospitals. Rathore $e t a l^{29}$ argue that 'cardiovascular care is a financially attractive service for hospitals' that provides up to $35 \%$ of total revenue, revenue which helps subsidise other necessary but less lucrative services. The authors warn that establishment of regional STEMI centres would increase the gap 'between the cardiac 'haves' and the 'have nots.' Another article concludes that non-PCI capable hospitals are the 'most likely to suffer a significant financial impact with the development of such systems, and their very survival may be threatened. ${ }^{13}$ However, our study found that, even under the very worst-case scenario where all ACS in-patient revenue would be lost, average PRL for STEMI referral hospitals would remain under $2 \%$. Furthermore, our analyses of PRL for ACS reveal that cardiology revenue centres within these hospitals receive the overwhelming majority of their revenue from non-ACS patients. 
Table 5 PRLs resulting from hypothetical routine diversion of all ACS patients away from referral hospitals with low or no $\mathrm{PCl}$ capability: individual hospital results

\begin{tabular}{|c|c|c|c|c|c|c|}
\hline \multirow{2}{*}{$\begin{array}{l}\text { Summary level for community hospital } \\
\text { financial data }\end{array}$} & \multicolumn{6}{|c|}{ Annual PRL (\%) } \\
\hline & Minimum & Median & Mean & 90th centile & 99th centile & Maximum \\
\hline All hospitals $(n=112)$ & 0.21 & 1.49 & 1.68 & 2.77 & 4.87 & 5.83 \\
\hline \multicolumn{7}{|l|}{ Community location } \\
\hline Small urban/rural $(n=10)$ & 0.29 & 1.53 & 1.53 & 3.03 & 3.74 & 3.74 \\
\hline Small rural/large urban $(n=16)$ & 0.22 & 1.45 & 1.57 & 3.34 & 3.68 & 3.69 \\
\hline Medium metropolitan $(n=22)$ & 0.33 & 1.54 & 1.89 & 3.44 & 4.88 & 4.87 \\
\hline Major metropolitan ( $n=64)$ & 0.51 & 1.48 & 1.65 & 2.69 & 5.83 & 5.83 \\
\hline \multicolumn{7}{|l|}{$\mathrm{PCl}$ capability in 2006} \\
\hline None performed $(n=78)$ & 0.22 & 1.49 & 1.61 & 2.73 & 4.87 & 4.87 \\
\hline $1-11(n=26)$ & 0.67 & 1.38 & 1.67 & 3.09 & 4.69 & 4.69 \\
\hline $12-51(n=3)$ & 1.11 & 1.21 & 1.30 & 1.60 & 1.60 & 1.60 \\
\hline $52+(n=5)$ & 1.80 & 2.53 & 2.95 & 5.83 & 5.83 & 5.83 \\
\hline \multicolumn{7}{|l|}{ Patients with STEMI in 2006} \\
\hline $1-11(n=53)$ & 0.22 & 1.08 & 1.22 & 2.11 & 3.74 & 3.74 \\
\hline $12-51(n=51)$ & 0.59 & 1.84 & 1.90 & 2.73 & 4.87 & 4.87 \\
\hline $52+(n=8)$ & 1.79 & 2.89 & 3.25 & 5.83 & 5.83 & 5.83 \\
\hline \multicolumn{7}{|l|}{ Hospital revenue center ${ }^{*}$} \\
\hline Cardiology procedures† ( $n=99 / 100)$ & 0.00 & 5.40 & 7.11 & 14.42 & 29.88 & 33.74 \\
\hline Intensive or coronary care unit $(n=107 / 107)$ & 0.65 & 3.08 & 3.67 & 6.66 & 10.98 & 13.30 \\
\hline Radiology ( $n=112 / 112)$ & 0.04 & 1.10 & 1.14 & 1.88 & 2.85 & 2.89 \\
\hline Pharmacy $(n=112 / 112)$ & 0.08 & 1.56 & 1.79 & 3.24 & 5.77 & 6.45 \\
\hline \multicolumn{7}{|l|}{ Patient payment type* } \\
\hline Medicare patients $(n=112 / 112)$ & 0.25 & 1.95 & 2.12 & 3.59 & 5.53 & 6.51 \\
\hline Medicaid patients $(n=94 / 112)$ & 0.02 & 0.80 & 1.11 & 2.25 & 15.02 & 15.02 \\
\hline Commercial payer Patients $(n=109 / 112)$ & 0.02 & 0.82 & 1.16 & 2.51 & 5.01 & 5.33 \\
\hline Self-pay/under-insured patients ( $n=98 / 112)$ & 0.05 & 1.18 & 1.93 & 4.30 & 14.46 & 14.46 \\
\hline Charity patients ( $n=52 / 79)$ & 0.24 & 1.53 & 2.42 & 5.65 & 10.32 & 10.32 \\
\hline Other $(n=65 / 110)$ & 0.02 & 0.98 & 1.64 & 4.26 & 7.46 & 7.46 \\
\hline \multicolumn{7}{|c|}{$\begin{array}{l}\text { *For each revenue center and payment type, in the labels below the numerator=the \# of hospitals with non-zero charges for patients with } \\
\text { STEMI and the denominator=the \# of hospitals with non-zero charges for all patients. } \\
\text { †Cardiology procedures include angiocardiography, cardiac catheterisation laboratory, cardiology, EKG and ECG. } \\
\text { ACS, acute coronary syndrome; PCl, percutaneous coronary intervention; PRL, projected revenue loss; STEMI, ST-elevation myocardial } \\
\text { infarction. }\end{array}$} \\
\hline
\end{tabular}

Our findings are consistent with other studies that have examined the financial impact of regionalisation on community hospitals. For example, Chappel et $a \ell^{30}$ concluded that rural hospitals would not suffer significant losses in procedure volume or revenue if specialised surgical procedures, including angioplasty, were diverted to high-volume hospitals. Another study estimated that low-volume rural PCI hospitals in Iowa would lose a combined $\$ 10.1$ million in revenue if patients were regionalised to high-volume hospitals. ${ }^{31}$ However, the Iowa study characterised 'low volume' hospitals as any hospital performing < 400 PCIs annually, much higher than our cut-off point of 200. Additionally, in our study, the highest PRLs were observed in those referral hospitals in medium and major metro areas $(\$ 0.40$ and $\$ 0.33$, respectively), not from those in rural locations.

Within hospitals, cardiology procedures and intensive or coronary care unit revenue centres experienced the

Table 6 Florida referral hospitals with $>1 \%$ PRL from proposed STEMI patient diversions/rapid transfers $(n=5)$

\begin{tabular}{|c|c|c|c|c|c|c|c|}
\hline $\begin{array}{l}\text { Hospital } \\
\text { ID }\end{array}$ & $\begin{array}{l}\text { County } \\
\text { location }\end{array}$ & $\begin{array}{l}\text { Distance to } \\
\text { nearest high } \\
\text { volume PCl } \\
\text { center (miles) }\end{array}$ & $\begin{array}{l}\text { Travel time to } \\
\text { nearest high } \\
\text { volume PCI } \\
\text { center (min) }\end{array}$ & $\begin{array}{l}\text { Number } \\
\text { of STEMI } \\
\text { inpatients } \\
\text { in } 2006 \\
\end{array}$ & $\begin{array}{l}\text { Per cent of } \\
\text { STEMIs who } \\
\text { received PCI }\end{array}$ & $\begin{array}{l}\text { STEMI } \\
\text { PRL (\%) }\end{array}$ & $\begin{array}{l}\text { Reported } \\
\text { profit margin } \\
\text { in } 2005(\%)\end{array}$ \\
\hline Hospital A & Major metro & 14 & 31 & 96 & $51 \%(n=49)$ & 3.82 & -11 \\
\hline Hospital B & Medium metro & 16 & 26 & 57 & $14 \%(n=8)$ & 1.68 & -10 \\
\hline Hospital C & Major metro & 8 & 16 & 98 & 0 & 1.19 & -6 \\
\hline Hospital D & Major metro & 9 & 18 & 93 & $89 \%(n=83)$ & 1.04 & +17 \\
\hline Hospital E & Medium metro & 11 & 17 & 25 & 0 & 1.03 & -21 \\
\hline
\end{tabular}


highest PRLs. Not surprisingly, cardiology procedures units showed the highest statewide PRL of any group, reaching $\$ 8.93 / \$ 100$ of revenue for all patients with ACS, but only $\$ 2.27$ for patients with STEMI. The PRLs in intensive or coronary care units reached $\$ 3.45$ per $\$ 100$ of revenue for patients with ACS. However, this figure drops to $\$ 0.59 / \$ 100$ for patients with STEMI.

Still, the prevailing characterisation of cardiac services as key profit-makers is based largely on historically favourable Medicare reimbursement rates, which in recent years have helped fuel a proliferation of specialty cardiac hospitals in many states. ${ }^{32}$ In our study, the majority of patients $(62 \%)$ were covered by Medicare. Total PRL for this group per $\$ 100$ of STEMI charges was only $\$ 0.36$, although this was slightly higher than the PRL for commercial payers $(\$ 0.30)$ and Medicaid (\$0.23). When focusing on all patients with ACS, the PRL for Medicare reached $\$ 2.24$, more than twice as high as that for commercial payers $(\$ 1.06)$. However, Medicare reimbursement rates for cardiac services were reduced in 2009, ${ }^{33}$ suggesting future PRL for Medicare patients will be even lower than our prediction. Of note, a recent study found that hospital financial strain caused by reduced reimbursement rates did not negatively affect the quality or timeliness of care offered to patients with AMI, regardless of patient insurance status. ${ }^{34}$

For both STEMI and ACS as a whole, PRLs were greatest for charity and self-pay/underinsured patients. Hospitals are required by law to treat these patients when they present to the ED, regardless of insurance status. However, given that in reality hospitals may collect very little revenue for these patients, diversion or rapid transfer of these patients would likely result in a net financial benefit for the referral hospital.

Critics have argued that the success of regional systems of transfer for PCI in Europe, for example as in Romania, ${ }^{35}$ cannot be generalised to the USA, where transfer distances tend to be much longer. However, regional systems in North Carolina and Minnesota have achieved promising results, even with patients in rural areas who have had to be transported over considerable distances. ${ }^{36}{ }^{37}$ The total costs and benefits of implementing such systems within the USA should be examined more closely to facilitate policy decisions.

One limitation of our study is that we were unable to account for the impact of routine STEMI transfer on patient costs. A system of transfer could result in added financial burdens for patients if they end up receiving care from physicians who do not participate in their insurance network. ${ }^{18}$ Such a system would need to establish protocols to prevent undue financial obligations for patients. Furthermore, a system of routine transfer of patients with STEMI would need to ensure all participating hospitals are rewarded for achieving efficient transfer times. A recent study in one hospital discovered that while achieving reduced door-to-balloon time decreased costs, it also resulted in lower payments. Consequently, the financial benefits of achieving greater efficiency and quality of care accrued solely to payers in this instance. ${ }^{38}$ Fenter $e t a l^{18}$ recommend establishing a system where a single payment is shared among 'the referring, transporting and receiving providers', so as to encourage coordination rather than competition between providers.

Our study population of patients with STEMI was defined on the basis of ICD-9-CM diagnosis codes, which may have resulted in a small degree of misclassification bias in our study.

Despite the advantages of early PCI for treating STEMIS, the costs of creating PCI capability in non-PCI capable hospitals can be prohibitive. Moreover, while expanding PCI capability to those hospitals that already have catheterisation laboratories has been shown to be feasible, ${ }^{39}$ this strategy will be likely to increase geographic access only minimally. ${ }^{40}$ Ultimately, a coordinated system of care for STEMIs will need to rely on both referral and receiving hospitals. A successful system can implement safeguards to protect the revenue of non-PCI capable hospitals. For example, the Boston EMS routinely transports patients with STEMI to PCI-capable hospitals, but its point of entry plan classifies these hospitals as 'STEMI hospitals' and PCI-referral hospitals as 'cardiac'. This helps ensure non-PCI capable hospitals continue to receive non-STEMI cardiac patients. ${ }^{41}$

STEMI referral hospitals are likely to continue to play a key role in treating STEMIs even if routine protocols for transfer to STEMI-receiving hospitals are implemented. Some patients with STEMI will have contraindications for PCI, making transfer unnecessary. In situations where patients presenting to a non-PCI capable hospital cannot undergo PCI within $120 \mathrm{~min}$ of first medical contact, treatment with fibrinolytics remains the recommended therapy unless contraindicated. ${ }^{19}$ Finally, there may be therapeutic and economic advantages in using facilitated PCI, or using PCI following fibrinolytic therapy. Coleman et at $t^{2}$ assert that major adverse cardiac end points and hospital costs were reduced significantly in facilitated versus primary PCI for transferred patients. Importantly, though, randomised controlled trials have not conclusively demonstrated advantages to facilitated PCI. ${ }^{43}$ Regardless of the treatment option, the future success of a system of routine transfer of patients to PCI-capable facilities will depend on mutually beneficial collaboration between hospitals that de-emphasises aggressive competition for revenues.

In conclusion, in this study we focused solely on the financial detriments to implementing regional systems of STEMI care in the USA. There are many other types of barriers to widespread adoption of STEMI transfer protocols. Transfer travel times are one important potential barrier. ${ }^{39}$ In Florida, a recent study in Florida found that the majority of potential STEMI referral hospitals were capable of transferring patients within guidelinerecommended time frames. ${ }^{44}$ However, in other regions of the USA, rapid transfer may not always be possible.

Finally, future research is needed to confirm the longterm cost-savings and patient benefits from STEMI 
systems of care from a system-wide or national perspective. In the UK, the National Health Service found that "PCI appears to be more cost effective than thrombolysis for people with AMI." ${ }^{45}$ The National Infarct Angioplasty Project in the UK estimated a net benefit of 333.5 million pounds from the implementation of a national system with routine transfer for PCI. ${ }^{46}$

\section{Twitter Follow Meg Comins at @mmcomins}

Acknowledgements The authors would like to acknowledge Jean Paul Tanner and Gabriella Anic for their assistance with preparation of the hospital transfer travel time database for Florida.

Contributors EBP and JAS conceived the study. All authors contributed to data analyses and report writing.

Funding This study was funded by a Grant-in-Aid (085574E) from the American Heart Association, Southeast Affiliate (Dr. Pathak, PI).

\section{Competing interests None declared.}

Provenance and peer review Not commissioned; externally peer reviewed.

Data sharing statement The data used in this study are publicly available from the Florida State Agency for Health Care Administration.

Open Access This is an Open Access article distributed in accordance with the Creative Commons Attribution Non Commercial (CC BY-NC 4.0) license, which permits others to distribute, remix, adapt, build upon this work noncommercially, and license their derivative works on different terms, provided the original work is properly cited and the use is non-commercial. See: http:// creativecommons.org/licenses/by-nc/4.0/

\section{REFERENCES}

1. Mozaffarian D, Benjamin E, Go A, et al. Executive summary: heart disease and stroke statistics-2015 update: a report from the American Heart Association. Circulation 2015;131:434-41.

2. Antman EM, Anbe DT, Armstrong PW, et al. ACC/AHA guidelines for the management of patients with ST-elevation myocardial infarction; a report of the American College of Cardiology/American Heart Association Task Force on Practice Guidelines (Committee to Revise the 1999 Guidelines for the Management of patients with acute myocardial infarction). Circulation 2004;110:588-636.

3. Andersen HR, Nielsen TT, Vesterlund T, et al., NAMI-2 Investigators. Danish multicenter randomized study on fibrinolytic therapy versus acute coronary angioplasty in acute myocardial infarction: rationale and design of the DANish trial in Acute Myocardial Infarction-2 (DANAMI-2). Am Heart $J$ 2003;146:234-41.

4. Andersen HR, Nielsen TT, Rasmussen $\mathrm{K}$, et al., DANAMI-2 Investigators. A comparison of coronary angioplasty with fibrinolytic therapy in acute myocardial infarction. $N$ Engl J Med 2003;349:733-42.

5. Brodie BR, Hansen C, Stuckey TD, et al. Door-to-balloon time with primary percutaneous coronary intervention for acute myocardial infarction impacts late cardiac mortality in high-risk patients and patients presenting early after the onset of symptoms. J Am Coll Cardiol 2006;47:289-95.

6. Keeley EC, Hillis LD. Primary PCI for myocardial infarction with ST-segment elevation. N Engl J Med 2007;356:47-54.

7. Magid DJ, Wang Y, Herrin J, et al. Relationship between time of day, day of week, timeliness of reperfusion, and in-hospital mortality for patients with acute ST-segment elevation myocardial infarction. JAMA 2005;294:803-12.

8. Widimsky P, Budesinsky T, Vorac D, et al., 'PRAGUE' Study Group Investigators. Long distance transport for primary angioplasty vs immediate thrombolysis in acute myocardial infarction. Final results of the randomized national multicentre trial-PRAGUE-2. Eur Heart J 2003;24:94-104.

9. Widimsky P, Bilkova D, Penicka M, et al. Long-term outcomes of patients with acute myocardial infarction presenting to hospitals without catheterization laboratory and randomized to immediate thrombolysis or interhospital transport for primary percutaneous coronary intervention. Five years' follow-up of the PRAGUE-2 trial. Eur Heart J 2007;28:679-84.
10. Keeley EC, Grines CL. Primary percutaneous coronary intervention for every patient with ST-segment elevation myocardial infarction: what stands in the way? Ann Intern Med 2004;141:298-304.

11. Jacobs AK, Antman EM, Ellrodt G, et al. AHA Consensus Statement: recommendation to develop strategies to increase the number of ST-segment-elevation myocardial infarction patients with timely access to primary percutaneous coronary intervention. Circulation 2006;113:2152-63.

12. Jacobs AK, Antman EM, Faxon DP, et al. Development of systems of care for ST-elevation myocardial infarction patients: executive summary. Circulation 2007;116:217-30.

13. Ellrodt G, Sadwin LB, Aversano T, et al. Development of systems of care for ST-elevation myocardial infarction patients: the non-percutaneous coronary intervention-capable (ST-elevation myocardial infarction referral) hospital perspective. Circulation 2007;116:e49-54.

14. Granger CB, Henry TD, Bates WE, et al. Development of systems of care for ST-elevation myocardial infarction patients: the primary percutaneous coronary intervention (ST-elevation myocardial infarction-receiving) hospital perspective. Circulation 2007;116:e55-9.

15. Moyer P, Ornato JP, Brady WJ Jr, et al. Development of systems of care for ST-elevation myocardial infarction patients: the emergency medical services and emergency department perspective. Circulation 2007;116:e43-8.

16. Sanz M, Smalling RW, Brewer DL, et al. Development of systems of care for ST-elevation myocardial infarction patients: the physician perspective. Circulation 2007;116:e39-42.

17. Mensah GA, Hand MM, Antman EM, et al. Development of systems of care for ST-elevation myocardial infarction patients: the patient and public perspective. Circulation 2007;116:e33-8

18. Fenter T, Golash T, Jensen N. Development of systems of care for ST-elevation myocardial infarction patients: the payer perspective. Circulation 2007;116:e60-3.

19. Levine GN, Bates ER, Blankenship JC, et al. 2011 ACCF/AHA/SCA guideline for percutaneous coronary intervention: a report of the American College of Cardiology Foundation/American Heart Association Task Force on Practice Guidelines and the Society for Cardiovascular Angiography and Interventions. Circulation 2011;124 e574-651.

20. Bednár F, Widimský P, Groch L, et al., PRAGUE-1 Study Group Investigators. Acute myocardial infarction complicated by early onset of heart failure: safety and feasibility of interhospital transfer for coronary angioplasty. Subanalysis of Killip II-IV patients from the PRAGUE-1 study. J Interv Cardiol 2003;16:201-8.

21. Bednár F, Widimský P, Krupicka J, et al., PRAGUE Study Group Investigators. Interhospital transport for primary angioplasty improves the long-term outcome of acute myocardial infarction compared with immediate thrombolysis in the nearest hospital (one-year follow-up of the PRAGUE-1 study). Can J Cardiol 2003;19:1133-7.

22. Kristensen SD, Andersen HR, Thuesen L, et al. Should patients with acute ST elevation MI be transferred for primary PCl? Heart 2004;90:1358-63.

23. Blankenship JC, Scott TD, Skelding KA, et al. Door-to-balloon times under $90 \mathrm{~min}$ can be routinely achieved for patients transferred for ST-segment elevation myocardial infarction percutaneous coronary intervention in a rural setting. J Am Coll Cardiol 2011;57:272-9.

24. American Hospital Association. American hospital association guide. Chicago, IL: Health Forum, 2007.

25. Economic Research Service. U.S. Department of Agriculture. 2004. http://www.ers.usda.gov/data-products/rural-urban-continuum-codes. aspx

26. Smith SC Jr, Feldman TE, Hirshfeld JW Jr, et al., ACC/AHA/SCAI 2005 guideline update for percutaneous coronary intervention: a report of the American College of Cardiology/ American Heart Association Task Force on Practice Guidelines (ACC/AHA/SCAI Writing Committee to Update the 2001 Guidelines for Percutaneous Coronary Intervention). Circulation 2006;113:e166-286.

27. Taira DA, Seto TB, Siegrist R, et al. Comparison of analytic approaches for the economic evaluation of new technologies alongside multicenter clinical trials. Am Heart J 2003;145:452-8.

28. U.S. Centers for Medicaid and Medicare Services. Description of Critical Care Access Hospitals. http://www.cms.gov/Medicare/ProviderEnrollment-and-Certification/CertificationandComplianc/CAHs.html

29. Rathore SS, Epstein AJ, Nallamothu BK, et al. Regionalization of ST-segment elevation acute coronary syndromes care: putting a national policy in proper perspective. J Am Coll Cardiol 2006;47:1346-9.

30. Chappel AR, Zuckerman RS, Finlayson SR. Small rural hospitals and high-risk operations: how would regionalization affect surgical volume and hospital revenue? J Am Coll Surg 2006;203:559-604. 
31. Ward MM, Jaana M, Wakefield DS, et al. What would be the effect of referral to high-volume hospitals in a largely rural state? $J$ Rural Health 2004;20:344-54.

32. Hayes KJ, Pettengil J, Stensland J. Getting the price right: medicare reimbursement rates for cardiovascular services. Health Aff 2007;26:124-36.

33. American College of Cardiology. Cardiology takes legal action against Medicare. 29 December 2009. http://www. campaignforpatientaccess.org/assets

34. Volpp KG, Konetzka RT, Zhu J, et al., National Registry of Myocardial Infarction Investigators. Effect of cuts in Medicare reimbursement on process and outcome of care for acute myocardial infarction patients. Circulation 2005;112:2268-75.

35. Benedek I, Gyongyosi M, Benedek T. A prospective regional registry of ST-elevation myocardial infarction in Central Romania: impact of the stent for life initiative recommendations on patient outcomes. Am Heart J 2013;166:457-65.

36. Henry TD, Sharkey SW, Burke N, et al. A regional system to provide timely access to percutaneous coronary intervention for ST-elevation myocardial infarction. Circulation 2007;116:721-8.

37. Jollis JG, Roettig ML, Aluko AO, et al., Reperfusion of Acute Myocardial Infarction in North Carolina Emergency Departments (RACE) Investigators. Implementation of a statewide system for coronary reperfusion for ST-segment elevation myocardial infarction. JAMA 2007;298:2371-80.

38. Khot UN, Johnson-Wood ML, Geddes JB, et al. Financial impact of reducing door-to-balloon time in ST-elevation myocardial infarction: a single hospital experience. BMC Cardiovasc Disord 2009;9:32.
39. Redberg RF. Reconsidering transfer for percutaneous coronary intervention strategy: time is of the essence. Arch Intern Med 2012;172:98-9.

40. Faxon D. Development of systems of care for ST-elevation myocardial infarction patients: current state of ST-elevation myocardial infarction care. Circulation 2007;116:e29-31.

41. Moyer P, Feldman J, Levine J, et al. Implications of the mechanical (PCI) vs thrombolytic controversy for ST segment elevation myocardial infarction on the organization of emergency medical services: the Boston experience. Crit Path Cardiol 2004;3:53-61.

42. Coleman $\mathrm{Cl}$, McKay RG, Boden WE, et al. Effectiveness and cost-effectiveness of facilitated percutaneous coronary intervention compared with primary percutaneous coronary intervention in patients with ST-segment elevation myocardial infarction transferred from community hospitals. Clin Ther 2006;28:1054-62.

43. Ellis SG, Tendera M, de Belder MA, et al. Facilitated $\mathrm{PCl}$ in patients with ST-elevation myocardial infarction. N Engl J Med 2008;358:2205-17.

44. Pathak EB, Forsyth CJ, Anic G, et al. Transfer travel times for primary percutaneous coronary intervention from low-volume and non-percutaneous coronary intervention-capable hospitals to high-volume centers in Florida. Ann Emerg Med 2011;58:257-66.

45. Hartwell D, Colquitt J, Loveman E, et al. Clinical effectiveness and cost effectiveness of immediate angioplasty for acute myocardial infarction: systematic review and economic evaluation. Health Technol Assess 2005;9:1-99, iii-iv.

46. National Infarct Angioplasty Project-Department of Health, UK. Impact assessment of treatment of heart attacks-national guidance. 2008. http://www.dh.gov.uk 